\title{
Sources of Loss Processes in Phonon Generation and Detection Experiments with Superconducting Tunneling Junctions
}

\author{
H.J. Trumpp and W. Eisenmenger \\ Universität Stuttgart, Physikalisches Institut, \\ Stuttgart, Federal Republic of Germany \\ Received August 11, 1977
}

\begin{abstract}
Observing the phonon yield, i.e. the ratio of the experimental phonon signal amplitude and the corresponding calculated value, phonon losses within the generation-detection system can be localized and determined quantitatively. With tin junctions on pure silicon substrates immersed in liquid helium the phonon yield is 3-5\%. Under vacuum conditions the yield rises to $10-12 \%$ indicating strong phonon transmission to the helium bath. The experimental lifetime for $280 \mathrm{GHz}$ phonons in the silicon substrate is longer than $65 \mu$ s indicating negligible volume losses and losses at the free substrate surface. It is further shown, that volume losses inside the phonon generator and detector are small compared to the total loss of about $90 \%$. By phonon reverberation measurements we find evidence that the main sources for phonon losses are localized at the boundaries of the tunneling junctions to the substrate. This is supported by an increase of the phonon yield with improved polishing from about $9 \%$ (mechanical), $10 \%$ (chemical) to $12 \%$ (sputter etching). A SIMS analysis indicates the presence of carbonhydrates and probably of water in the boundaries. This layer of extraneous molecules together with the nonideal surface structure of the substrate and the evaporated films weakens the mechanical bonding between the tunnel junctions and the substrate and is possibly causing strong phonon splitting by anharmonic forces.
\end{abstract}

\section{Introduction}

Phonon generation and detection experiments with superconducting tunneling junctions [1-3] give information on the polarization dependent electronphonon interaction strength, $2 \Delta$-phonon trapping in superconductors [4-6], phonon escape into liquid helium and solids [4-8] and the emitted phonon spectrum [9-14].

In first quantitative generation-detection experiments $[7,8]$ we measured $2 \Delta$-phonon losses of $95 \%$ to $98 \%$ having the sample in liquid helium contact. This result raised the question of the nature of the loss processes involved or alternatively of the correctness of the theoretical models used for the evaluation. During propagation phonons can interact with quasiparticles, lattice defects, impurities, isotopes and thermal phonons or undergo spontaneous splitting. In order to determine the phonon losses quantitatively, to localize them and to investigate their physical nature we calculated recombination phonon signal amplitudes for three independent experimental situations and corresponding models and compared them with the result of measurements [15]. All experiments and calculations in this work refer to 24 recombination phonons which are detected by a superconducting tunneling junction if the phonon frequency is equal to or higher than the detector energy gap. The quotient of the experimental signal amplitude and the corresponding calculated value is called phonon yield. The models contain assumptions for phonon generation, emission, propagation and detection. By variation of the experimental parameters as: orientation of the crystal, perpendicular or oblique emission, measurement with helium contact or under vacuum conditions, and taking account of phonon focusing in the crystal [16-20], we have been able to check the intensity distribution for the emitted phonons and the strength of the phonon emission into the helium bath and into the substrate crystal.

In Section 2 we discuss the three different experimental situations and the corresponding models used to derive the phonon yield. Section 3 describes experi- 
mental data, sample preparation techniques, the low temperature apparatus and some aspects of the pulse techniques used for phonon generation and signal averaging. Experimental results as the phonon transmission into the liquid helium, the $2 \Delta$-phonon lifetime in dielectric crystals and the phonon yield data are presented in Section 4. These results are then discussed with respect to our theoretical model assumptions for calculating the phonon yield and with respect to the possible phonon loss sources.

\section{Models for Calculating Phonon Signals}

\subsection{Ballistic Model}

In most cases the sample consists of two superconducting tin tunneling junctions which are vacuum deposited on the opposite faces of cylindrical silicon crystals. The ballistic model is a calculation of the signal caused by $2 \Delta$-phonons which propagate within the geometrical aperture angle $\varphi_{D}$ ballistically to the detector and break up Cooper-pairs. From generation to detection no phonon losses are taken into account. Therefore, the $2 \Delta$-phonon loss $K_{B}$ is expressed through the ballistic phonon yield $\alpha_{B}\left(\alpha_{B}\right.$ =quotient of the experimental and the calculated signal):

$K_{B}=1-\alpha_{B}$.

All experimental data required to determine the ballistic phonon yield $\alpha_{B}$ can be measured having the sample immersed in liquid helium or under vacuum conditions.

A superconducting tunneling junction biased at $2 \Delta / e \leqq U_{G} \leqq 4 \Delta / e$ generates one $2 \Delta$-recombination phonon per tunneling electron [1]. $I_{G}$ being the tunneling current, the generated $2 \Delta$-phonon rate is

$\dot{n}=I_{G} / e$.

As phonon propagation inside the generator is concerned, the microcrystalline film structure is taken as elastically isotropic expressed by two sound velocities for transverse and longitudinal phonons $c_{t}, c_{l}$. Phonons with energies $\Omega \geqq 2 \Delta$ are reabsorbed [4-6] by Cooper-pair breaking within the mean free path $A_{w}$ $\left(A_{w} \approx 1000 \AA\right.$ for tin) corresponding to a reabsorption time constant $\tau_{w}=A_{w} / c_{t, l} \approx 5 \cdot 10^{-11} \mathrm{~s}$. The intrinsic quasiparticle recombination time constant $\tau_{R}$ is inversely proportional to the thermal equilibrium quasiparticle density $N_{T}$ which for $T<0.5 T_{c}$ is approximately [3]:

$N_{T}=2 N_{0}(2 \pi \Delta k T)^{1 / 2} \exp (-\Delta / k T)$
$N_{0}=$ density of electron states per spin at the Fermi level. Typical values of $\tau_{R}$ for tin at $T=1 \mathrm{~K}$ are $\tau_{R} \approx 10^{-8} \mathrm{~s}$; since $\tau_{w} \approx 5 \cdot 10^{-11} \mathrm{~s}$ the comparison shows that the $2 \Delta$-energy exists mainly in form of quasiparticles but can only leave the tunneling junction as phonons. Reabsorption leads to a retarded decay of an excess quasiparticle density i.e. prolongs the intrinsic recombination lifetime $\tau_{R}$ to the effective lifetime $\tau_{\text {eff }}$ directly measured in pulse decay experiments. In tin junctions of $d=4000 \AA$ thickness on silicon in vacuum at $T=1 \mathrm{~K}$ appr. 150 reabsorption events take place before a phonon is emitted to the substrate $[4,5] . \tau_{\text {eff }}$ is mainly limited by the junction thickness and the acoustic boundary conditions for phonon emission to the adjacent media. 24-phonon losses in the junction volume by interaction with quasiparticles, defects, recombination under emission of photons or under emission of two phonons $<24$ and recombination over a reduced gap through trapped magnetic flux are small compared to the $2 \Delta$ phonon emission in the case of tin, for $T<T_{c} / 2$ and pure films oriented parallel to the earth-magnetic field. A ray acoustic phonon trapping model [4] shows a linear thickness dependence of $\tau_{\text {eff }}$ for $A_{w}<d \ll A_{v}, A_{v}$ being the mean free path for volume losses:

$\tau_{\text {eff }}=d \frac{N_{T}}{N_{\omega T}}\left(\frac{1}{c_{t}^{3}}+\frac{1}{2 c_{l}^{3}}\right)\left(\frac{\bar{T}_{t}}{c_{t}^{2}}+\frac{\bar{T}_{l}}{2 c_{l}^{2}}\right)^{-1}$

where $N_{\omega T}$ is the $2 \Delta$-phonon density in thermal equilibrium, $c_{t l}$ the polarization dependent sound velocity and $\bar{T}_{t l}$ the integral phonon transmission to the adjacent helium and substrate $\bar{T}_{t l}=\bar{T}_{t l, \mathrm{He}}+\bar{T}_{t l, \mathrm{~S}}$ :

$\bar{T}_{t l}=\int_{0}^{\varphi_{t l, g}} T_{t l}(\varphi) \sin 2 \varphi d \varphi$

$T_{t l}(\varphi)=$ angle dependent phonon transmission, $\varphi_{t l, g}$ $=$ angle of total reflection $\left(\varphi_{\mathrm{g}}=90^{\circ}\right.$ in the medium with the higher sound velocity, $\varphi_{\mathrm{g}}<90^{\circ}$ in the medium with the lower sound velocity). Experimental values $\tau_{\text {eff }}(d)$ with tin junctions on silicon substrates [5] show the linear thickness dependence in agreement with the calculated slope in the range $3000 \AA<d<8000 \AA$ :

$\tau_{\text {eff,exp }}=7 \cdot 10^{-2} \frac{d}{\mathrm{~cm}}[\mathrm{~s}]$

$\tau_{\text {eff, calc }}=6.8 \cdot 10^{-2} \frac{d}{\mathrm{~cm}}[\mathrm{~s}]$

at $T=1.02 \mathrm{~K}, \Delta_{\mathrm{Sn}}=590 \mu \mathrm{eV}$.

This agreement also confirms that $A_{v} \gg d$, i.e. negligible volume losses in the detector and generator. The integral transmission to the substrate $\bar{T}_{\mathrm{Sn} / \mathrm{Si}}$ and to the helium bath $\bar{T}_{\mathrm{Sn} / \mathrm{He}}$ can be determined by inserting 
the experimental slope $\tau_{\text {eff }}(d)$ and the ratio $N_{T} / N_{\omega T}$ from detailed balance [4-6] into (4). Taking only account of the dominant transverse contribution to the transmission (appr. $85 \%$ ) we obtain:

$$
\bar{T}_{\mathrm{Sn} / \mathrm{Si}}+\bar{T}_{\mathrm{Sn} / \mathrm{He}}=\frac{d}{\tau_{\mathrm{eff}}} \frac{N_{T}}{N_{\mathrm{w} T}} \frac{1}{c_{t}} .
$$

The relative intensity of transverse to longitudinal 2A-phonons $A_{t} / A_{l}$ in the flux to the substrate boundary is completely determined by the phonon density of states since reabsorption changes primarily generated phonon occupations to quasithermal equilibrium according to

$\left(A_{t} / A_{l}\right)_{G}=2 c_{l}^{2} / c_{t}^{2}$.

Phonons incident at angles $\varphi$ within the cone of total reflection on the substrate boundary are transmitted with the probability $T(\varphi)$ and emitted into the full half space of the substrate with an angular intensity distribution following approximately Lambert's cosine law $[16,21]$. In addition, the intensity distribution is determined by phonon focusing [18] in anisotropic media. In the case of silicon, focusing enhances or reduces the intensity up to a factor of about ten. With increasing distance from the generator $r$, the intensity varies as $r^{-2}$ if $r$ exceeds the junction diameter $r \gg a$, assuming negligible volume losses in the substrate. Using (2) the calculated number of phonons incident on the detector per unit time $\dot{n}_{D}$, i.e. the phonon rate results in

$\dot{n}_{D}=I_{G} F_{D} \bar{f}_{\sigma, \varphi_{D}} / e \pi r_{G D}^{2}$.

$F_{D}=$ detector area $\bar{f}_{\sigma, \varphi_{D}}=$ mean focusing factor averaged over all phonon modes $\sigma$ and the geometrical aperture angle to the detector $\varphi_{D}, r_{G D}=$ distance generator-detector. Ballistic 24-phonon losses in the substrate crystal can occur by the decay in phonons with less energy, i.e. spontaneous splitting, thermal stimulated splitting, elastic and inelastic interaction with defects as isotopes, displacements and extraneous atoms. With data from references [22-24] an estimate of the mean free paths $\Lambda_{i}, \Lambda_{e}$, due to inelastic and elastic processes of pure dislocation free silicon at $T=1 \mathrm{~K}$ respectively, leads to $\Lambda_{i} \approx 300 \mathrm{~cm}$, $A_{e} \approx 40 \mathrm{~cm}$, i.e. negligible volume losses in the substrate at a typical distance generator-detector $r_{G D} \approx 1 \mathrm{~cm}$.

A significant signal parameter is also the ratio $A_{t} / A_{l}$ of the emitted transverse and longitudinal phonons which is changed as compared to (7) by the polarization dependent transmission $\bar{T}_{t l}$ and phonon focusing $\bar{f}_{t l, \varphi_{D}}$. Therefore, we expect a phonon signal amplitude ratio using (7)

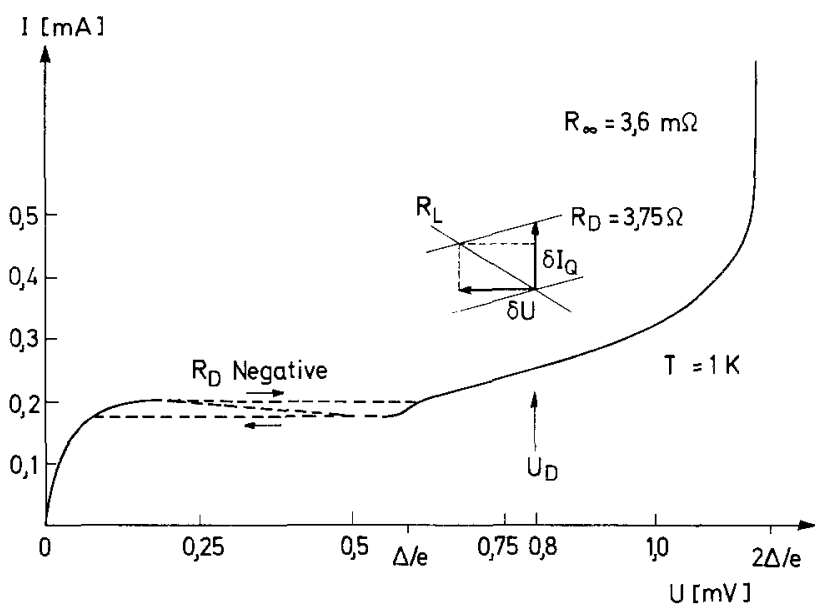

Fig. 1. $I(U)$ characteristic of a tin junction in the thermal tunneling current regime. Leakage current contributions are small compared to the thermal tunneling current. The insert elucidates the origin of the voltage signal $\delta U$

$A_{t} / A_{l}=\left(2 c_{l}^{2} / c_{t}^{2}\right)\left(\bar{T}_{t} / \bar{T}_{l}\right)\left(\bar{f}_{t, \varphi_{D}} / \bar{f}_{l, \varphi_{D}}\right)$.

The detector phonon rate $\dot{n}_{D}$ and the amplitude ratio $A_{t} / A_{l}$ is practically not changed by the transmission into the detector if determined by acoustic mismatch. The transmission for perpendicular incidence is:

$T_{\perp}=1-R_{\perp}=4 Z_{1} Z_{2} /\left(Z_{1}+Z_{2}\right)^{2}$

where $R_{\perp}$ is the reflection coefficient for perpendicular incidence and $Z=\rho c$ the characteristic impedance; $\rho=$ density. $T_{\perp}$ usually exceeds 0.95 ; in the case silicon/tin we have $T_{\perp}>0.99$ for both polarizations.

24-phonons are detected quantitatively by Cooperpair-breaking, if the mean free path for reabsorption $A_{w}$ is shorter than the detector film thickness and, as generally assumed, small compared to the mean free path against volume losses $A_{v}, A_{w}<d, A_{w} \ll A_{v}$ $\left(\Lambda_{w, \mathrm{Sn}} \approx 1000 \AA, d=3000 \AA\right)$. With respect to the neglection of volume losses and also of the signal contribution of low energy phonons, we find that the absorption of 24 -phonons by Cooper-pairs does well exceed the absorption by quasiparticles. For $T / T_{c}<0.5$ the ratio of superconducting to normal state absorption is $\alpha_{S} / \alpha_{N}<0.05$ [25], showing negligible contributions of phonon scattering by quasiparticles. Other detection mechanisms, such as phonon assisted tunneling, AC-Josephson-effect and simultaneous detection of two or more phonons with energies $\Omega<2 \triangle_{D}$, are weak compared to the dominant pairbreaking by single phonon detection.

The operation of the detector is illustrated in Figure 1. Usually a detector is biased in the thermal tunneling current regime at $U_{D}=4 \Delta / 3(0.8 \mathrm{meV}$ for tin) where $I(U)$ is linear. DC-Josephson currents are suppressed by a magnetic field of 10-20 Gauß. 
Thermally excited quasiparticles $N_{T}$ [4] give rise to a thermal tunneling current $I_{T}\left(U_{D}\right)$

$$
I_{T}=\left(2 e R_{\infty}\right)^{-1}\left(N_{T} / N_{0}\right)\left(e U_{D}+\Delta\right)\left[\left(e U_{D}+\Delta\right)^{2}-\Delta^{2}\right]^{-1 / 2}
$$

$R_{\infty}=$ resistance for tunneling between normal conductors. Detection of $2 \Delta$-phonons at a rate $\dot{n}_{D}$ causes injection of two quasiparticles per phonon. The quasiparticle excitation rate per volume is

$\dot{N}_{Q}=2 \dot{n}_{D} / V_{D}$

$V_{D}=$ Detector volume, $V_{D}=F_{D} d$.

Constant injection enhances the thermal quasiparticle density

$N_{T}$ to $N_{T}+\delta N_{Q}$

$\delta N_{Q}=\dot{N}_{Q} \tau_{\text {eff }}$.

For the temperature dependence of the effective quasiparticle lifetime $\tau_{\text {eff }}$ we get with (3), (4) and $N_{\omega T} \sim T \exp (-2 \Delta / k T)[4]$

$\tau_{\text {eff }}=$ const. $T^{-1 / 2} \exp (\Delta / k T)$.

In the linear regime of phonon detection $\delta N_{Q} \ll N_{T}$, the excess quasiparticle density $\delta N_{Q}$ produces the short circuit signal current $\delta I_{Q}$. With (11) we have

$$
\delta I_{Q}=\left(2 e R_{\infty}\right)^{-1}\left(\delta N_{Q} / N_{0}\right)(e U+\Delta)\left[(e U+\Delta)^{2}-\Delta^{2}\right]^{-1 / 2}
$$

and

$\delta I_{Q} / I_{T}=\delta N_{Q} / N_{T}$.

In pulse experiments, as performed in this work, the detector biasing point $U_{D}$ is held at constant current, and $\delta I_{Q}$ is measured in terms of the open circuit voltage change $\delta U$. From [3] or the insert of Figure 1 $\delta I_{Q}$ results in

$\delta I_{Q}=-\delta U\left(R_{D}^{-1}+R_{L}^{-1}\right)$

$R_{D}=$ dynamic detector resistance at the biasing point. $R_{L}=$ load resistance of the detector circuit given by the resistance of the pulse transformer on the side of the detector $R_{L}=4 \Omega$. Transformer and detector are connected by $4 \Omega$ striplines. On the side of the preamplifier the transformer resistance was $50 \Omega$.

The voltage signal $\delta U$ follows from the amplified signal voltage at the recorder (or oscilloscope) $U_{S}$ divided by the total voltage amplification factor $A$ of the pulse transformer, preamplifier, boxcarintegrator (or sampling unit)

$\delta U=U_{S} / A$

$A$ is measured by superimposing a pulse signal of known height on the junction and comparison with the amplified pulse height. From the experimental signal in saturation (pulse width $P \gg \tau_{\text {eff }}$ ) the ballistic contribution $U_{B}$ is extracted (i.e. contributions from sidewall reflection subtracted). The ratio of the experimental ballistic signal current to the generator current is using $(17,18)$ :

$\left(I_{B} / I_{G}\right)_{\text {exp }}=-\left(U_{B} / I_{G} A\right)\left(R_{D}^{-1}+R_{L}^{-1}\right)$.

For large phonon signals, nonlinearities in the detector $I(U)$-characteristic near the biasing point forbid the use of (17) without corrections. Besides this, nonlinear phonon detection occurs for $\delta N \gg N_{T}$. This regime of overinjection with $\delta I_{Q} \sim \dot{n}_{D}^{1 / 2}[6,26]$ is, therefore, also called the "square root limit" of phonon detection. If $\delta N \geqq N_{T}$, pulse decay is not exponential and $\tau_{\text {eff }}$ is reduced to a dynamic decay time [6]. To avoid complicated corrections, experiments are usually performed in the linear regime of small phonon signals $\delta N_{Q}<N_{T}$ where $\tau_{\text {eff }}$ is directly obtained from the exponential pulse decay. For a correct measurement of the effective quasiparticle lifetime, $\tau_{\text {eff }}$ must exceed the electronic detector time constant $\tau_{e}[3]$

$\tau_{e}=C_{D} R_{D} R_{L} /\left(R_{D}+R_{L}\right)$

$C_{D}=$ capacitance of the detector junction (with $R_{D} \approx R_{L}=1 \Omega, R_{\infty}=10^{-3} \Omega, C_{D}$ is about $10^{-8}$ Farad and $\tau_{e} \approx 10 \mathrm{~ns}$; whereas $\tau_{\text {eff, Sn, } 1 \mathrm{~K}}>100 \mathrm{~ns}$ ). The signal current $\delta I_{Q}$ is now replaced by the ballistic signal $I_{B}$, and from (8), (12), (13), (16) and $V_{D}=F_{D} d$ we obtain the calculated ratio of the ballistic signal current and the generator current with:

$\left(I_{B} / I_{G}\right)_{\mathrm{cal}}=2 I_{T} \tau_{\mathrm{eff}} f_{\sigma, \varphi_{D}} / e \pi N_{T} d r_{G D}^{2}$.

In the case of oblique emission or/and detection (21) has to be multiplied with the cosine of the corresponding angles $\beta_{G}, \beta_{D}$

$\left(I_{B} / I_{G}\right)_{\mathrm{cal}}=\left(2 I_{T} \tau_{\mathrm{eff}} f_{\sigma, \varphi_{D}} / e \pi N_{T} d r_{G D}^{2}\right) \cos \beta_{G} \cos \beta_{D}$.

Dividing (19) by (21) the ballistic phonon yield is:

$\alpha_{B}=\left(I_{B} / I_{G}\right)_{\text {exp }} /\left(I_{B} / I_{G}\right)_{\mathrm{cal}}$

$=\frac{e \pi\left(-U_{B}\right)\left(R_{D}^{-1}+R_{L}^{-1}\right)}{2} \frac{N_{T}}{I_{G} A} \frac{d}{I_{T} A f} \frac{r_{G D}^{2}}{f_{\sigma, \varphi_{D}}}$.

We note that $N_{T} / I_{T}$ in (23) can be replaced by the quantities $R_{\infty}, N_{0}$ and $U_{D}$. Equations (3) and (11) result in

$N_{T} / I_{T}=2 e R_{\infty} N_{0}\left[(e U+\Delta)^{2}-\Delta^{2}\right]^{1 / 2} /(e U+\Delta)$.

In the direct use of $I_{T}$ possible additional current contributions as leakage current and two particle 


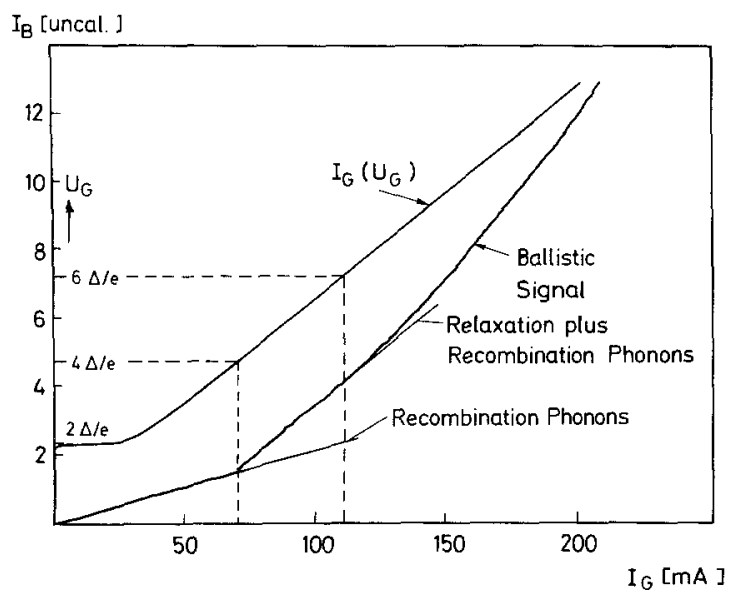

Fig. 2. Dependence of the ballistic signal $I_{B}$ on the generator current $I_{G}$ for tin junctions on a silicon crystal at $T=1.01 \mathrm{~K}$

tunneling current have to be subtracted by extrapolating the temperature dependence of $I_{T}\left(U_{D}\right)$ to $T$ $=0 \mathrm{~K}$.

Figure 2 shows the dependence of the ballistic signal $I_{B}$ from the generator current $I_{G}$ for tin junctions on a silicon substrate. At generator voltages $0<$ $U_{\mathrm{G}}<4 \Delta / e$ the detection of $2 \Delta$-recombination phonons leads to a linear slope of $I_{B}\left(I_{G}\right)$ which can be used to determine the experimental signal ratio $\left(I_{B} / I_{G}\right)_{\text {exp }}$. The sharp signal gradient change at $U_{G}$ $=4 \mathrm{~A} / \mathrm{e}$ originates from the detection onset of relaxation phonons with energies $\Omega \geqq 2 \Delta$ [1-4]. It proves negligible contributions of relaxation phonon energies $\Omega<2 \Delta$ to the signal, i.e. the signal results exclusively from detection of phonons with energies $\Omega \geqq 2 \Delta$. Thus, by observing the $4 \Delta$ gradient change, each signal contribution used for the determination of the phonon yield was checked to arise from 24 phonons.

\subsection{Reverberation Model}

In addition to the direct ballistic signal contributions 24-phonons are emitted in the full half space into the crystal volume. These phonons undergo one or multiple sidewall reflections before being detected. With generator pulsewidth longer than the phonon lifetime, therefore, a stationary density of diffusely propagating $2 \Delta$-phonons can be generated in the crystal volume. For the calculation of the detector signal produced by these $2 \Delta$-phonons we use a reverberation model.

Because of the anomalous small Kapitza resistance to the helium bath, reverberation signals are observed only under vacuum conditions. In vacuum, however, the reverberation signal can well exceed the ballistic signal height depending on sample purity, orientation, volume, surface area covered by superconducting films and the mechanical contact to the sample holder etc. The experimental $2 \Delta$-phonon lifetime in the crystal $\tau_{\mathrm{C}}$ or the reverberation time, is a measure for the corresponding phonon losses. Through mode conversion by phonon reflection at the free crystal surface [21] the experimental lifetime is the effective common lifetime for all phonon modes. Like $\tau_{\text {eff }}, \tau_{C}$ is obtained from pulse decay measurements.

In calculating the stationary reverberation signal we assume absence of phonon losses in the generator junction and in the phonon emission into the substrate. Using (2) the stationary $2 \Delta$-phonon density $n_{C}$ in the crystal volume for constant phonon generation at a rate $\dot{n}_{G}$ results in

$n_{C}=\dot{n}_{G} \tau_{C} / V_{C}=I_{G} \tau_{C} / e V_{C}$

$V_{C}=$ crystal volume. Since $\tau_{C}$ is the common lifetime for all phonon modes, we calculate the $2 \Delta$-phonon flux to the detector with a mean sound velocity $\bar{c}\left(\bar{c}_{\mathrm{Si}}\right.$ $=5.5 \mathrm{~km} / \mathrm{s}[19,20]$ ). The substrate medium is treated as elastically isotropic. This appears appropriate with respect to the reverberation signal, since from symmetry considerations focusing effects can be shown to average out over the half space. For diffuse phonon incidence the effective detector area is

$F_{\text {eff }}=F_{D} / 4$

With (25) the $2 \Delta$-reverberation phonon rate transmitted to the detector can be expressed by

$\dot{n}_{R}=I_{G} \tau_{C} \bar{c} F_{\text {eff }} \bar{T}_{C D} / e V_{C}$

where $\bar{T}_{C D}=\int_{0}^{\pi / 2} T(\varphi) \sin 2 \varphi d \varphi$ is the transmission from the crystal to the detector $\left(\bar{T}_{\mathrm{S} / \mathrm{Sn}} \approx 0.9\right)$. Using (12), (13), (16), (27), the same arguments as in the derivation of the ballistic signal lead to a calculated ratio of the reverberation signal and the generator current

$\left(\frac{I_{R}}{I_{G}}\right)_{\mathrm{cal}}=\frac{2 \tau_{\mathrm{eff}} I_{T}}{e N_{T}} \frac{\bar{c} \tau_{C}}{V_{C}} \frac{F_{\mathrm{eff}} \bar{T}_{C D}}{V_{D}}$

With (26) and the detector thickness $d=V_{D} / F_{D}(28)$ can be written:

$\left(\frac{I_{R}}{I_{G}}\right)_{\mathrm{cal}}=\frac{\tau_{\mathrm{eff}} I_{T}}{2 e N_{T}} \frac{\bar{c} \tau_{C}}{V_{C}} \frac{\bar{T}_{C D}}{d}$.

From (19) results the experimental value $\left(I_{R} / I_{G}\right)_{\exp }$ and the reverberation phonon yield is: 


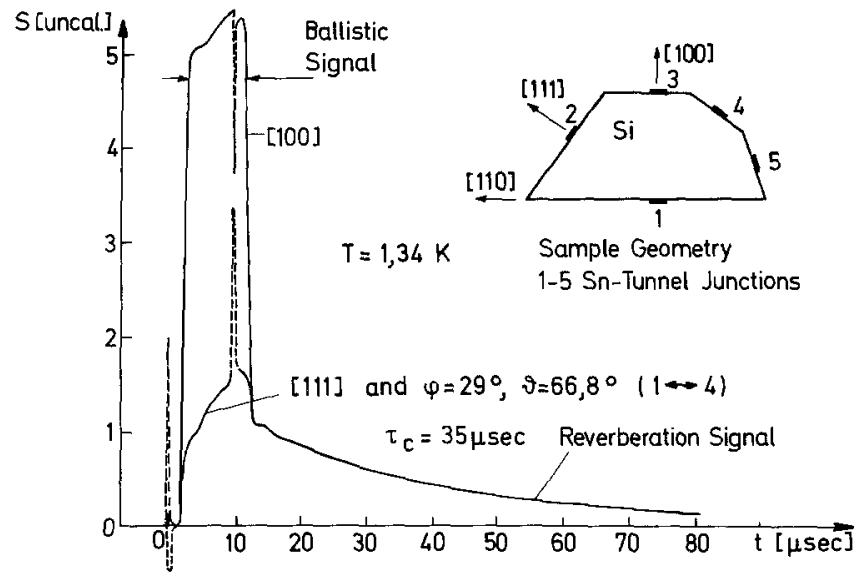

Fig. 3. Direct comparison of ballistic signal amplitudes in vacuum for different propagation directions. The corresponding reverberation signal amplitude is independent of the detector position. The evaporation surfaces are mechanically polished. Sample geometry see insert. Pulsewidth $10 \mu \mathrm{s}, \varphi_{D}=5^{\circ}, r_{G D}=1 \mathrm{~cm}$.

$$
\begin{aligned}
& \alpha_{R}=\left(I_{R} / I_{G}\right)_{\mathrm{exp}} /\left(I_{R} / I_{G}\right)_{\mathrm{cal}} \\
& =\frac{-U_{R}}{I_{G} A}\left(R_{D}^{-1}+R_{L}^{-1}\right) \frac{2 e N_{T} V_{C} d}{\tau_{\mathrm{eff}} I_{T} \bar{c} \tau_{\mathrm{C}} \bar{T}_{C D}}
\end{aligned}
$$

where $U_{R}$ is the reverberation voltage signal amplified by a factor $A$ defined by (18). Ballistic and reverberation signal contributions are shown in Figure 3. In contrast to the ballistic model, the reverberation model does not depend on the shape of the generator emission characteristic or on the phonon focusing. Moreover, the reverberation phonon loss $K_{R}=1-\alpha_{R}$ contains only losses in the generator volume and at the interface between the junctions and the substrate, but not within the substrate, since $\left(I_{R} / I_{G}\right)_{c a l}$ is calculated using the experimental 2A-phonon lifetime in the crystal $\tau_{C}$. Thus, $K_{B}-K_{R}$ is a measure for $2 \Delta$ phonon losses in the crystal volume due to inelastic and elastic interactions.

\subsection{Dominant Boundary Loss Model}

Our reverberation experiments in vacuum indicate a strong linear dependence of the reciprocal $2 \Delta$-phonon lifetime in the crystal on the surface covered by metal films $F_{F}$ divided by the crystal volume $V_{C}$. The resulting decay rate $\tau_{C}^{-1}=$ const. $F_{F} / V_{C}$ can be called a phonon splitting rate. The agreement for $\tau_{\text {eff }}(d)$ in experiment and theory as discussed in Section 2.1 proves the loss at the film covered surface to be a boundary loss and not a volume loss in the superconductor.

With the 2A-phonon lifetime for volume losses in the crystal $\tau_{V}$, the lifetime for losses at the free substrate

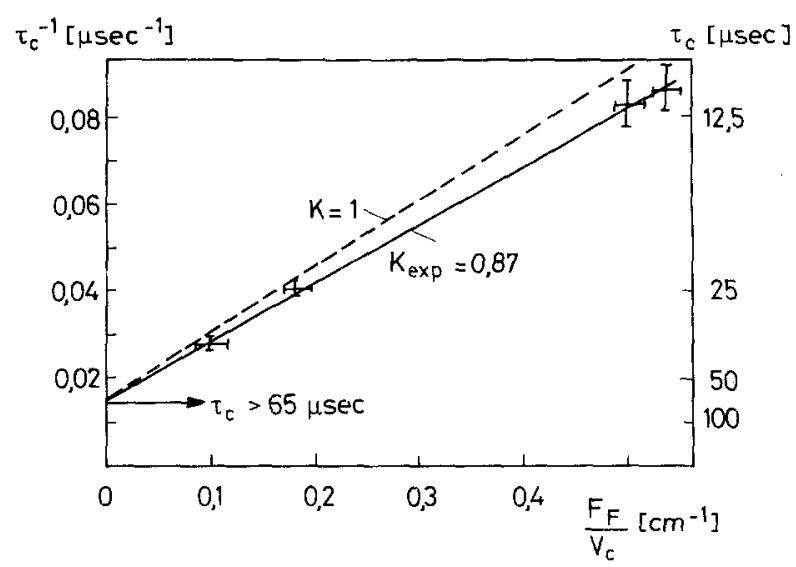

Fig. 4. Dependence of the 2A-phonon lifetime in the crystal on the ratio of the film covered surface and the crystal volume. The slope with $K=1$ is calculated from (31)

surface $\tau_{S}$ and at the film covered surface $\tau_{F}, \tau_{C}$ can be written:

$\tau_{C}^{-1}=\tau_{V}^{-1}+\tau_{S}^{-1}+\tau_{F}^{-1}=\tau_{V}^{-1}+\tau_{S}^{-1}+K_{F} F_{F} \vec{c} / 4 V_{C}$

$K_{F}=$ splitting probability of $2 \Delta$-phonons for impact at the film covered surface (absorption plus reemission).

For dominant loss at the $\mathrm{Sn}-\mathrm{Si}$ boundary, $K_{F}$ is equivalent to the ballistic and the reverberation phonon loss $K_{B}, K_{R}$ since phonons traverse the boundary twice by absorption plus reemission as well as in generation - detection experiments. The phonon yield $\alpha_{F}=1-K_{F}$ is determined from experiment on the basis of (31) using the graphical presentation of $\tau_{c}^{-1}\left(F_{F}\right)$ data as in Figure 4. Agreement and differences in the 2A-phonon yield obtained with the three models $\alpha_{B}, \alpha_{R}, \alpha_{F}$ allow the localization of phonon losses.

\section{Experimental Data and Procedure}

The substrates are in most cases cylindrical silicon crystals of $15 \mathrm{~mm} \varnothing$ and a length of $6-20 \mathrm{~mm}$. They are orientated in 100,110 or in the 111-direction $\pm 0.5^{\circ}$, the actual ballistic phonon propagation direction generator - detector. One crystal has a facet cut geometry (see insert of Fig. 3) allowing direct comparision of phonon signal height and yield in different orientations as well as oblique emission and/or detection. In this way phonon focusing, the validity of Lambert's cosine law for phonon emission and eventually orientation dependent phonon thermalization processes can be proved or corrected. The silicon is 
$\leqq 10^{12} / \mathrm{cm}^{3}$ boron-doped and dislocation free, $E P D$ $=0$. After sawing and lapping the crystals are chemically polished (CP6, Schäfer-etch) to remove surface dislocations. This is followed by mechanical polishing of the evaporation surfaces with diamond grain, the diameter stepwise decreased to $0.25 \mu$. In some cases, this is followed by chemical etch-polishing or $d c$-sputter-polishing using a Veeco microetch argon ion gun (rotating sample, $15^{\circ}$ grazing incidence, $\mathrm{Ar}$ energy $1000 \mathrm{~V}-300 \mathrm{~V}$, etch rate $2-10 \AA / \mathrm{s}$ ).

After sputtering, the junction films are deposited without breaking the evaporator vacuum. This is of advantage compared to mechanical and chemical polishing since the thickness of oxide and extraneous molecule layers is reduced. As generator and detector we used tin junctions of $d=3000 \AA-5000 \AA$ thickness and $\leqq 1 \mathrm{~mm}^{2}$ overlap area. One experiment was performed using an aluminium junction as detector to prove the influence of a reduced detector gap on the phonon yield. The films were vacuum deposited at a pressure of appr. $5 \cdot 10^{-6}$ Torr and a rate of $5 \AA / \mathrm{s}$ to $140 \AA / \mathrm{s}$. A glow discharge was used for the fabrication of the oxigen barrier. During oxidation it is convenient to screen the sample from the luminous part of the discharge by metallic shields $\left(10^{-1}\right.$ Torr const. $\mathrm{O}_{2}$-pressure, $-1000 \mathrm{~V}$ against ground, $t=$ $8 \mathrm{~min})$. In some experiments the junctions were evaporated at a slight oxigen background of $5 \cdot 10^{-6}$ Torr $\mathrm{O}_{2}$ to ameliorate the junction quality, i.e. the ratio of the maximum single particle tunneling current at $U=2 \mathrm{~A} / e$ and of the leakage current $I_{24} / I_{L}$. Additional improvement was accomplished by cooling the substrate to appr. $275 \mathrm{~K}$ during film deposition. Typical asymptotic tunneling resistances $R_{\infty}$ for the tin junctions range from $2 \cdot 10^{-3} \Omega$ to $10^{-1} \Omega$ with quality ratios $I_{2 \Delta} / I_{L}>100$.

Using a $\mathrm{He}^{4}$ glass cryostate plus pumping system temperatures of $1 \mathrm{~K}$ are reached for $6-8 \mathrm{~h}$. The sample is inside a vacuum chamber surrounded by liquid $\mathrm{He}^{4}$ with the possibility to perform the experiments having the entire sample immersed in $\mathrm{He}$ or under vacuum conditions. This choice allows the determination of the phonon transmission to the Hebath $\bar{T}_{\mathrm{He}}$ and to the crystal $\bar{T}_{C}$, as discussed in Section 2.1. Besides this, vacuum conditions are necessary to measure the $2 \Delta$-phonon lifetime in the crystal $\tau_{C}$ and the reverberation signal (compare Sect. 2.2).

The experiments are performed in pulse technique to measure lifetimes directly by pulse decay and to resolve phonon signal contributions of different velocity. Especially in vacuum experiments pulsed conditions are advantageous to avoid sample heating. In measurements of saturated signal contributions the pulse width $P$ must exceed the longest lifetime of phonons or quasiparticles by which the signal height is determined $P>\tau . P<\tau$ is convenient for $\tau$-measurements to elude pulse decay deformations and to use the correct baseline for evaluation. The electrical aspects of pulse measurements are discussed in Section 2.1.

\section{Experimental Results and Conclusions}

\subsection{Short Pulse Signals}

Figure 5 shows a mode resolved pulse signal of $280 \mathrm{GHz}$ phonons propagating ballistically in the 111-silicon direction with contributions of sidewall reflected phonons. The amplitude ratio of transverse to longitudinal phonons is $A_{t} / A_{l}=3.8$. In comparing amplitude ratios $A_{t} / A_{l}$ using (9) with experimental ratios for different orientations and detector aperture angles $\varphi_{D}$, the strength of phonon focusing can be tested (see Table 1). For the main directions 100,110 and 111 the calculated and experimental $A_{t} / A_{l}$-values agree within a factor two. Moreover, the $\varphi_{D^{-}}$ dependence of the $A_{t} / A_{l}$ ratios in the 111-direction is verified by experiment especially for mechanical polish with $\varphi_{D}=3^{\circ}, 5^{\circ}$ and $7.5^{\circ}$. In 111-direction the differences in $A_{t} / A_{t}$ between experiment and calculation, depending mainly on polish, are appr. $-30 \%$ for mechanical and sputter polish and appr. $+100 \%$ for chemical polish. As will be discussed in Section 4.4 , the phonon yield $\alpha_{B}$, which is mainly given by transverse signal contributions, depends not as much on polish. This indicates enhanced splitting of longitudinal phonons in the junction-substrate interface for chemically polished silicon crystals. For the case of mechanical and sputter polish, it appears that the phonon thermalization in the boundary is of about the same magnitude for longitudinal and transverse phonons.

Experimental $A_{t} / A_{t}$-ratios under helium conditions exceed vacuum values by $5 \%$ to $20 \%$ indicating that the signal ratio is influenced by the emission of phonons from the generator to the helium bath, although 2 24 -phonons are reabsorbed in the superconducting film within the mean free path $A_{w}<d$ ( $d$ =film thickness appr. $4000 \AA, \Lambda_{w, \mathrm{Sn}_{\mathrm{n}}} \approx 1000 \AA$ ). This can be understood assuming that the mean free path for longitudinal phonons in $\mathrm{Sn}$ is about twice as high as for transverse phonons $\Lambda_{w, l}=2 \Lambda_{w, t}=2000 \AA$. Depending on evaporation conditions and film thickness, tin films are usually rough. Minimum and maximum thicknesses of $1000 \AA$ and $5000 \AA$, respectively, at an average thickness of $d=3000 \AA$ are possible. In this way only the emission of longitudinal phonons to the helium can cause a change in $A_{t} / A_{t}$-values since strong reabsorption of transverse phonons prohibits 


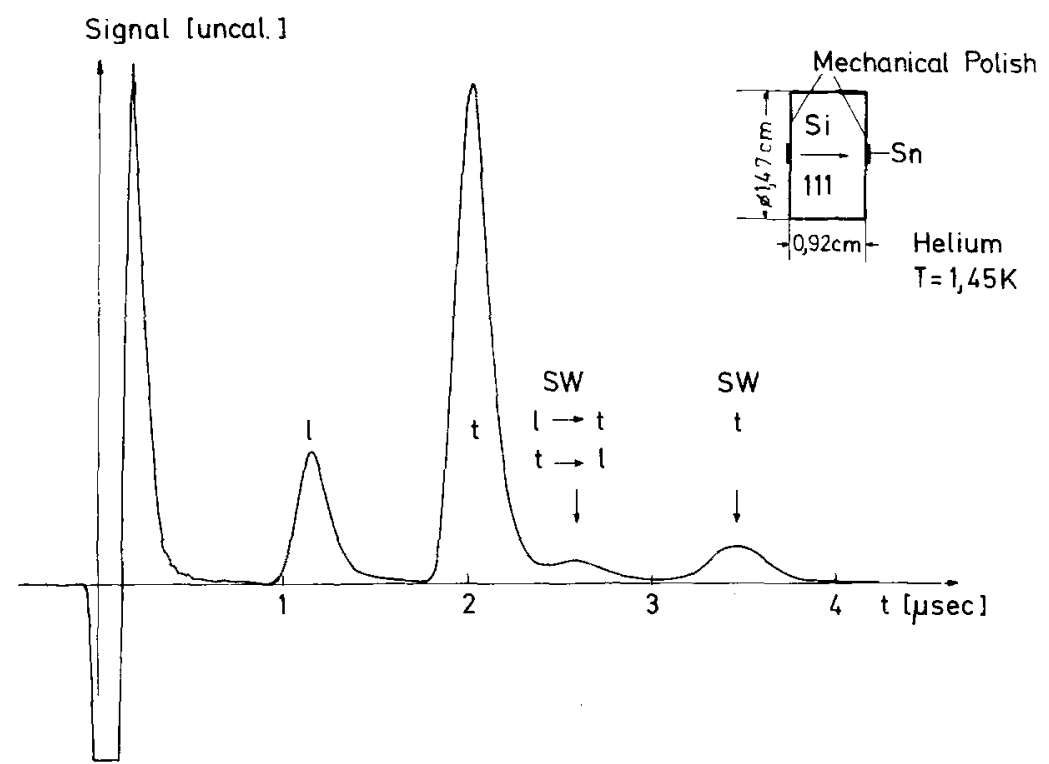

Fig. 5. Short pulse signal in 111-direction. Besides ballistic signal contributions a sidewall reflected transverse and a mode converted contribution can be seen direct emission to the detector after previous reflection at the tin-helium interface.

From pulse decay in short pulse signals at temperatures $T \approx 1 \mathrm{~K}$ for tin the effective quasiparticle lifetime $\tau_{\text {eff }}$ can be measured. As discussed in Section 2.1 the integral phonon transmission to the helium $\bar{T}_{\mathrm{He}}$ and to the substrate $\bar{T}_{S}$ is given by inserting $\tau_{\text {eff }}$ for helium and vacuum conditions $\left(\bar{T}_{\mathrm{He}}=0\right)$ in (6) [7]. From detailed balance $[4,6]$ the quotient $N_{T} / N_{\omega T}$ is 1430 for tin at $1 \mathrm{~K}$. With the experimental slope from [5] $\tau_{\text {eff }, V}=7 \cdot 10^{-2} \mathrm{~d} / \mathrm{cm}, \tau_{\text {eff }, \text { He }}=1.7 \cdot 10^{-2} \mathrm{~d} / \mathrm{cm}$ (6) results in $\bar{T}_{\mathrm{Sn} / \mathrm{Si}}=0.12$ being in fair agreement with $\bar{T}_{\mathrm{Sn} / \mathrm{Si}}=0.0937$ [27] calculated from acoustic mismatch. In contrast to the transmission to the substrate, the transmission to the helium $\bar{T}_{\mathrm{Sn} / \mathrm{He}}=0.32$ \pm 0.5 exceeds the calculated value $\bar{T}_{\mathrm{Sn} / \mathrm{He}}<0.01$ significantly. The enhanced transmission is in accord with the reduced Kapitza resistance found in heat conduction experiments [28]. So far no satisfactory explanation is known for the physical mechanism of the Kapitza resistance [16, 17, 28-33] reduction.

The linear dependence $\tau_{\text {eff }}(d)$ in [5] has been observed for tin junctions evaporated without oxygen background. As discussed in Sections 2.1, the linear thickness dependence $\tau_{\text {eff }}(d)$ indicates negligible volume losses in the tin films. Evaporation of the tin films with an oxigen background pressure of appr. $5 \cdot 10^{-6}$ Torr (sample in vacuum) results in $\tau_{\text {eff }}$ reduced to $60 \%$ of the oxygen free value. From detailed balance equations this reduction indicates vol-

Table 1. Comparison of experimental $A_{t} / A_{l}$-values with calculated values using (9). From (7) the generated ratio is $\left(A_{t} / A_{t}\right)_{G}=7.95$. Snjunctions on Si substrates. $\mathrm{He}=$ helium contact, $V=$ vacuum; $\mathrm{mp}, \mathrm{cp}$, ip = mechanical, chemical and sputter polish; ft, st $=$ fast and slow transverse phonons. Error in the experimental $A_{t} / A_{l}$-values: $111 \pm 5 \%, 110 \pm 10 \%, 100 \pm 20 \%$

\begin{tabular}{|c|c|c|c|c|c|c|c|c|c|c|c|}
\hline \multirow{2}{*}{\multicolumn{3}{|c|}{$\begin{array}{l}\text { Propagation } \\
\text { direction and } \\
\text { detector aperture } \\
\text { angle } \varphi_{D}\end{array}$}} & \multirow[t]{2}{*}{$\begin{array}{l}\bar{T}_{t} / \bar{T}_{l} \\
\text { Eq. }(5), \varphi_{D}<10^{\circ}\end{array}$} & \multirow[t]{2}{*}{$\begin{array}{l}\vec{f}_{\sigma, \varphi_{D}} \\
\text { Lit. [19] }\end{array}$} & \multirow{2}{*}{\multicolumn{2}{|c|}{$\begin{array}{l}\bar{f}_{t, \varphi_{\mathbf{D}}} / \bar{f}_{l, \varphi_{D}} \\
\text { Lit. [19] }\end{array}$}} & \multirow[t]{2}{*}{$\begin{array}{l}\left(A_{t} / A_{l}\right)_{\mathrm{cal}} \\
\mathrm{Eq} .(9)\end{array}$} & \multicolumn{4}{|c|}{$\left(A_{t} / A_{l}\right)_{\exp }$} \\
\hline & & & & & & & & $\mathrm{He}$ & $\varphi_{D}$ & $V$ & $\varphi_{D}$ \\
\hline \multirow[t]{6}{*}{111} & \multirow{6}{*}{$\varphi_{D}$} & \multirow[t]{2}{*}{$8^{\circ}$} & \multirow[t]{6}{*}{0.82} & \multirow[t]{2}{*}{2.3} & \multirow{2}{*}{\multicolumn{2}{|c|}{1.0}} & \multirow[t]{2}{*}{6.5} & $4.0 \mathrm{mp}$ & $7.5^{\circ}$ & $3.8 \mathrm{mp}$ & $7.5^{\circ}$ \\
\hline & & & & & & & & $3.8 \mathrm{mp}$ & $5^{\circ}$ & $3.6 \mathrm{mp}$ & $5^{\circ}$ \\
\hline & & \multirow[t]{2}{*}{$5^{\circ}$} & & \multirow[t]{2}{*}{2.2} & \multirow{2}{*}{\multicolumn{2}{|c|}{0.84}} & \multirow[t]{2}{*}{5.5} & $3.1 \mathrm{mp}$ & $3^{\circ}$ & $2.5 \mathrm{mp}$ & $3^{\circ}$ \\
\hline & & & & & & & & $3.5 \mathrm{ip}$ & $9^{\circ}$ & $3.0 \mathrm{ip}$ & $9^{\circ}$ \\
\hline & & \multirow[t]{2}{*}{$3^{\circ}$} & & \multirow[t]{2}{*}{2.0} & \multicolumn{2}{|l|}{0.70} & 4.6 & $14.2 \mathrm{cp}$ & $8^{\circ}$ & $13.8 \mathrm{cp}$ & $8^{\circ}$ \\
\hline & & & & & $\mathrm{ft}$ & st & & & & & \\
\hline \multirow[t]{3}{*}{110} & \multirow[t]{3}{*}{$\varphi_{D}$} & $8^{\circ}$ & $0.627 \mathrm{ft}$ & 3.3 & 2.33 & 0.3 & 11.6 & & & \multirow[t]{3}{*}{$11 \mathrm{mp}$} & \multirow[t]{3}{*}{$9^{\circ}$} \\
\hline & & $5^{\circ}$ & $0.97 \mathrm{st}$ & 3.5 & 2.86 & 0.27 & 14.2 & & & & \\
\hline & & $3^{\circ}$ & & 4.0 & 3.85 & 0.25 & 19.0 & & & & \\
\hline \multirow[t]{3}{*}{100} & \multirow[t]{3}{*}{$\varphi_{D}$} & $8^{\circ}$ & 0.525 & 6.2 & \multirow{3}{*}{\multicolumn{2}{|c|}{$\begin{array}{l}18 \\
16.75 \\
14\end{array}$}} & 75 & $70 \mathrm{ip}$ & $7.5^{\circ}$ & 70 ip & $7.5^{\circ}$ \\
\hline & & $5^{\circ}$ & & 5.8 & & & 70 & $70 \mathrm{mp}$ & $5^{\circ}$ & $70 \mathrm{mp}$ & $5^{\circ}$ \\
\hline & & $3^{\circ}$ & & 5.0 & & & 58 & & & & \\
\hline
\end{tabular}


ume losses of $2 \Delta$-phonons up to $30 \%$ of the generated phonons. In He-measurements the $\tau_{\text {eff }}$ reduction of $\mathrm{O}_{2}$-doped junctions is appr. $20 \%$. This could partially be caused by enhanced transmission to the helium since by $\mathrm{O}_{2}$-perturbation the grain diameter is reduced with a possible increase of the film surface area.

Experimentally the theoretical temperature dependence $\tau_{\text {eff }} \sim T^{-1 / 2} \exp (\Delta / k T)$ from (14) was verified down to $T=1.1 \mathrm{~K}$ independent from oxygen admixture. Below $1.1 \mathrm{~K}$ the increase of $\tau_{\text {eff }}$ with decreasing $T$ is limited by recombination at trapped magnetic flux, i.e. spots of reduced energy gap. In order to reduce this $\tau_{\text {eff }}$ saturation it is convenient to orientate the junctions parallel to the earth magnetic field. For $2 \Delta$-phonon generation in tin tunneling junctions this production of phonons $<2 \Delta$ is usually negligible, since recombination is so fast by overinjection that quasiparticles do not "find" spots of trapped magnetic flux within their lifetime limited diffusion path.

\subsection{Ballistic and Reverberation Signal with Long Generator Pulses}

In the case of phonon generation with long pulses, i.e. pulsewidth exceeding the time of flight, the contributions of different phonon modes to the ballistic signal add up to the stationary ballistic signal. In vacuum experiments the sidewall-reflected $2 \Delta$-phonon signal contributions likewise add up to the reverberation signal (see Fig. 3). Because of mode conversion for reflection at the free surface, the lifetime $\tau_{C}$ of the $2 \Delta$-phonons in the substrate crystal is the common lifetime of all phonon modes, as discussed in Section 2.2. Both the ballistic and the reverberation signal were checked to arise from detection of $2 \Delta$-phonons (compare Sect. 2.1).

A silicon crystal with a facet cut geometry (see insert of Fig. 3) allows direct comparison of phonon signals for 111, 100 and other propagation directions. Thus, experimental focusing values can be collated with data derived from Elbaum [19], and Rösch, Weis [20]. Moreover, the validity of Lambert's cosine law for the emitted phonon intensity distribution has been checked by using different oblique angles of emission or detection. Figure 3 shows the $2 \Delta$-phonon signal of junction 1 as detector at a constant generator current $I_{G}=100 \mathrm{~mA}$ of the junctions 2,3 and 4 in turn. The relative ballistic signal heights are

$S_{100}(3 \rightarrow 1) / S_{111}(2 \rightarrow 1)=5.2$;

$S_{111}(2 \rightarrow 1) / S_{\varphi, \vartheta}(4 \rightarrow 1)=1$

$\left(\varphi=29^{\circ} ; \vartheta=66.8^{\circ}\right)$.
The reduced ballistic phonon signals, i.e. $S$ normalized by division with the focusing factor (averaged over the polarizations $\sigma$ and aperture angle $\varphi_{D}$, see Table 1) and the cosinus $\vartheta$-value agree in the three propagation directions:

$S_{100} / \bar{f}_{\sigma 100}=5.2 / 5.8=0.9$;

$S_{111} / \bar{f}_{\sigma 111} \cos 54.8^{\circ}=0.8$

$S_{\varphi, 2} / \bar{f}_{\sigma ; \varphi, \vartheta} \cos 35^{\circ}=1$.

Using in reverse directions junction 1 as generator and junctions 2,3 and 4 as detectors, the reduced ballistic signal heights are the same as above. The agreement of the reduced signal amplitudes is good, especially with regard to the tolerances of the focusing factors of $\pm 10 \%$. Therefore, the comparison of the different ballistic signal directions shows the validity of the focusing model and the cosine law for phonon emission.

In contrast to the ballistic signal the reverberation signal does not depend on the generator-substratedetector geometry, i.e. the experimental $2 \Delta$-phonon lifetime in the crystal $\tau_{C}$ is the same for detectors on different crystal planes (see Fig. 3). This means that focusing effects are averaged out over the half space in front of the detector, as discussed in Section 2.2. The reverberation time $\tau_{C}$ is temperature independent in the range $1.0 \mathrm{~K}<T<1.5 \mathrm{~K}$ indicating negligible contributions of thermally stimulated processes to the splitting of $2 \Delta$-phonons in the silicon crystals. The time constant $\tau_{C}=35 \mu \mathrm{sec}$ corresponds to a mean free path $A_{C}$ for 24 -phonons of $A_{C}=20 \mathrm{~cm}$ (crystal volume $V_{C}=3.4 \mathrm{~cm}^{3}$ ). Consequently, $280 \mathrm{GHz}$ phonons can undergo more than 10 reflections at the crystal surface within $\tau_{C}$. As consequence of mode conversion by phonon reflection, $\tau_{C}$ corresponds to an average of the inelastic decay rates for all polarizations and is limited by one or more phonon branches showing the fastest inelastic scattering processes. The magnitude of the $\tau_{C}$ values indicates the small contribution of volume losses to the signal reduction in phonon propagation experiments. Furthermore, phonon reflection at the free substrate surface apparently is predominantly elastic.

Reflection at the Sn film covered surface, however, as discussed in the following Section is mainly inelastic. Here reflection is understood in the sense of transmission from the crystal into the tin film and subsequent reemission into the crystal. The calculated integral acoustic transmission factor from silicon to tin is $\bar{T}=0.9$. The existence of boundary losses is found experimentally from the dependence of $\tau_{C}$ on the ratio of the tin film covered surface area and the crystal volume $\tau_{C}\left(F_{F} / V_{C}\right)$. 
Table 2. Ballistic and reverberation phonon yield for different sample parameters. The orientation is the actual ballistic propagation direction. $\alpha_{B, H e}$ is measured having the sample in helium contact. $V$ $=$ vacuum. Values obtained with tin junctions evaporated without $\mathrm{O}_{2}$-doping are underlined. $\mathrm{mp}, \mathrm{cp}$, ip $=$ see Table 1 . The two values of $\alpha_{B, \mathrm{He}}, \alpha_{B, \mathrm{r}}, \alpha_{R v}$ in each line correspond to direct and reverse phonon transmission. The phonon yield obtained with the dominant boundary loss model is $\alpha_{F}$ $=10-15 \%$ (see Fig. 4). Using an Al-junction as detector $\left(A_{\mathrm{Al}}=0.26 \mathrm{meV}\right)$ the ballistic phonon yield was $\alpha_{B, \mathrm{Sn} / \mathrm{Al}}=30 \%$

\begin{tabular}{|c|c|c|c|c|c|c|c|c|}
\hline Orientation & $r_{G, D}[\mathrm{~cm}]$ & Polish & $\alpha_{B, \mathrm{He}}$ & {$[\%]$} & \multicolumn{2}{|c|}{$\alpha_{B, V}[\%]$} & \multicolumn{2}{|c|}{$\alpha_{R, V}[\%]$} \\
\hline 111 & 0.915 & $\mathrm{mp}$ & $\underline{4.0}$ & $\underline{3.8}$ & & & & \\
\hline 111 & 0.76 & $\mathrm{mp}$ & $\underline{2.2}$ & 2.0 & $\underline{8.8}$ & 9.5 & & \\
\hline 111 & 1.98 & $\mathrm{mp}$ & 3.9 & 4.5 & 7.3 & 7.0 & 10.5 & 9.6 \\
\hline 111 & 0.75 & $\mathrm{cp}$ & $\underline{5.5}$ & $\underline{5.1}$ & & & & \\
\hline 111 & 0.68 & $\mathrm{cp}$ & 3.7 & & $\underline{9.0}$ & 10.0 & & \\
\hline 111 & 0.66 & ip & 6.0 & 4.4 & $1 \overline{0.6}$ & 9.0 & & \\
\hline 111 & 0.64 & ip & 7.6 & 4.0 & & & & \\
\hline 111 & 0.64 & ip & 4.8 & 3.0 & & & & \\
\hline 111 & 0.63 & ip & 4.1 & 4.4 & 13.0 & 14.0 & 10.0 & 11.0 \\
\hline 111 & 0.62 & ip & 6.5 & 7.0 & 12.4 & 13.2 & 16.0 & 17.0 \\
\hline 100 & 0.74 & ip & 7.5 & 6.5 & 13.0 & 12.0 & & \\
\hline 100 & 0.74 & ip & 3.5 & 2.8 & 9.6 & 8.0 & & \\
\hline 110 & 0.63 & $\mathrm{mp}$ & & & 13.6 & 13.3 & 11.5 & 12.0 \\
\hline
\end{tabular}

Table 3. Phonon yield $\alpha_{B}$ (ballistic) and $\alpha_{R}$ (reverberation) obtained with tin junctions evaporated on a silicon crystal of facet cut geometry (see insert of Fig. 3). Vacuum conditions, mechanical polish, $r_{G D}=1 \mathrm{~cm}$

\begin{tabular}{llcl}
\hline Orientation & Direction & $\alpha_{B}[\%]$ & $\alpha_{R}[\%]$ \\
\hline 111 & $1 \rightarrow 2$ & 10.0 & 11.0 \\
& $2 \rightarrow 1$ & 9.3 & 11.0 \\
100 & $1 \rightarrow 3$ & 9.0 & 12.0 \\
& $3 \rightarrow 1$ & 9.3 & 12.0 \\
$\varphi=29^{\circ}$ & $1 \rightarrow 4$ & 8.5 & 11.0 \\
$\vartheta=66.8^{\circ}$ & $4 \rightarrow 1$ & 9.3 & 11.0 \\
\hline
\end{tabular}

\subsection{Dependence of the Phonon Lifetime in the Crystal on the Tin Film Area}

Figure 4 shows a linear increase of the phonon splitting rate $\tau_{C}^{-1}$ as function of the ratio of the $\mathrm{Sn}$ film covered surface area and the crystal volume. The linearity indicates the dominance of phonon splitting at the film covered surface over thermalization processes at the free crystal surface and in the crystal volume. The slope of the $\tau_{C}^{-1}\left(F_{F} / V_{C}\right)$-function is used for the determination of the boundary loss $K_{F}$ or the corresponding phonon yield $\alpha_{F}$ as discussed in Section 2.3 and the following Section. Extrapolating the slope to a tin film area $F_{F}=0$ determines the lower limit of 2 $\Delta$-phonon lifetimes in the volume and at the surface. Using (31) and Figure 4 results in $\tau_{C}^{-1}=\tau_{V}^{-1}$ $+\tau_{s}^{-1}=1.5 \times 10^{-8} \mathrm{~s}^{-1}$, i.e. $\tau_{V}>65 \mu \mathrm{s}, \tau_{s}>65 \mu \mathrm{s}$ corresponding to a mean free path for inelastic scatter- ing of $A_{C}>35 \mathrm{~cm}$. From theoretical considerations $[22,23] \tau_{V}$ is $\geqq 10^{3} \mu$ s for longitudinal $280 \mathrm{GHz}$ phonons at $T=1 \mathrm{~K}$. Therefore, in our experiment $\tau_{C}$ may possibly be limited by $\tau_{S}\left(\tau_{V} \gg \tau_{S}\right)$. Experimental values of $\tau_{C}$ in Verneuil- $\mathrm{Al}_{2} \mathrm{O}_{3}$ crystals [34] amount to only $1 / 3$ of the silicon data measured at the same covering with tin.

\subsection{Results for the Phonon Yield}

The phonon yield was determined for all different experiments and found to be independent from temperature.

Tables 2 and 3 contain the corresponding data. In general the errors resulting from limited accuracy of the experimental data amount to $\pm 30 \%$. In a few cases error reduction by averaging was possible.

According to the strong transmission from the generator to the helium the values derived from the ballistic model under helium conditions amount to approximately one third of the vacuum values $\alpha_{B, \mathrm{He}}=2$ $7.5 \% ; \alpha_{B, V}=7-13.5 \%$. But even under vacuum conditions the $2 \Delta$-phonon loss shows the high value of $K_{B, V}=1-\alpha_{B, V} \approx 90 \%$. Different phonon propagation directions lead to a ballistic phonon yield of about the same value although the focusing factors vary considerably (see Table 1). This evidence of the validity of the focusing model is as convincing as following from the comparison of the ballistic signal amplitudes in Section 4.2. Moreover, the agreement in $\alpha_{B}$ for oblique emission or oblique detection in the 
111 direction of the facet cut crystal (see Fig. 3) affirms the cosine law for phonon emission.

In Section 2.1 we concluded from $\tau_{\text {eff }}(d)$-measurements negligible volume losses in pure tin films. Even in tin films evaporated with oxygen background volume losses amount only to a small fraction of the total loss (Sect. 4.1). There we found also that emission of undetectable phonons with reduced energy by recombination at trapped magnetic flux can be disregarded. But energy reduction could occur by overinjection in the generator. Although this seems unlikely, since $\alpha_{B}$ was independent of injection current and we used generators of considerably different tunneling resistance, we checked the influence of overinjection on $\alpha_{B}$ by detection with an aluminium junction $\Delta_{\mathrm{A} 1}=0.26 \mathrm{meV}, \Delta_{\mathrm{Sn}}=0.59 \mathrm{meV} . \alpha_{B}$ increased to $30 \%$, but not to $100 \%$, as to be expected if gap reduction in the generator was the main reason for the phonon loss. As can be shown, the observed finite yield enhancement arises from extra detection of plitted phonons.

Since the junctions generate and detect 24-phonons quantitatively, the loss may originate in principle as volume loss in the substrate or in the boundary to it. The reverberation yield $\alpha_{R}=10-16 \%$ is calculated with the experimental $2 \Delta$-phonon lifetime in the crystal $\tau_{C}$ and, therefore, does not contain any substrate volume losses. Hence it follows: the loss $K_{R}=1-\alpha_{R}$ is a boundary loss. Moreover, this conclusion can be drawn by the length of $\tau_{C}$ alone (compare Sect. 4.2). On an average the reverberation yield is $2 \%$ higher than the ballistic yield. On the supposition that the difference is caused by a volume loss we can calculate a volume lifetime $\tau_{V}$. Using $\Delta \alpha_{R, B}=\alpha_{R}-\alpha_{B}=0.02=1$ $-\exp \left(-t_{G, D} / \tau_{V}\right)$, time of flight between generator and detector $t_{G, D}=2 \mu \mathrm{s}$, we get $\tau_{V}=100 \mu \mathrm{s}$. This is about one order of magnitude smaller than the calculated value $\tau_{V} \geqq 1000 \mu$ s of Section 4.3. Possibly the reason for a finite $\Delta \alpha_{R, B}$ is elastic scattering by isotopes which reduces $\alpha_{B}$ but leaves $\alpha_{R}$ unaffected. From [24] we calculate a mean free path for elastic scattering by isotopes in silicon of $A_{i, \text { el }}=35 \mathrm{~cm}$ corresponding to $\tau_{i, \mathrm{el}}=60 \mu \mathrm{s}$, which is comparable to the observed $\tau_{V}$.

The linear dependence $\tau_{C}\left(F_{F} / V_{C}\right)$ in Figure 4 shows that the boundary loss is not due to mechanical or other surface defects but depends on the tin covered substrate area. That means, 2A-phonons partially split into two or more phonons of lower frequency while traversing the film-substrate boundary. The phonon yield $\alpha_{F}$ from the dominant boundary loss model (see Sect. 2.3) describes the 2A-phonon loss for transmission to tin and subsequent reemission. $\alpha_{F}$ is determined from the slope of $\tau_{C}^{-1}\left(F_{F} / V_{C}\right)$ to be $\alpha_{F}=1$ $-K_{F}=10-15 \%$ and is as high as $\alpha_{B}, \alpha_{R}$. Since also in ballistic generation-detection experiments the phonons traverse the boundary twice, we conclude from the agreement of the phonon yields and the smallness of volume losses in the Sn films and the $\mathrm{Si}$ crystal that the loss is dominantly a boundary loss.

For tin junctions on $\mathrm{Al}_{2} \mathrm{O}_{3}$ substrates the yield is lower than for silicon $\alpha_{B, \mathrm{He}}=1-2 \%$ [7]. Thus, we believe that the loss doesn't originate from the properties of silicon as semiconductor (Schottky barrier). This is also supported by experiments on phonon losses [35] at the interface of In on $\mathrm{Al}_{2} \mathrm{O}_{3}$.

Obviously it is important to obtain information on a possible frequency dependence of the strength of the loss mechanism. For different junction materials the phonon frequencies are: $v_{\mathrm{Al}}=85-140 \mathrm{GHz}$ (depending on the admixture of other materials, for example perturbation by oxygen), $v_{\mathrm{Sn}}=280-290 \mathrm{GHz}, v_{\mathrm{Pb}}=$ $870 \mathrm{GHz}$. Using silicon as substrate the corresponding ballistic yields in vacuum have been observed: $\alpha_{B, \mathrm{Al}}=18-25 \%[13] ; \alpha_{B, \mathrm{Sn}}=8-12 \% ; \alpha_{B, \mathrm{~Pb}}=3-4 \%$ [36]. Generation of $280 \mathrm{GHz}$ relaxation phonons in Al-junctions and detection by tin junctions yielded $\alpha_{B}=8-10 \%, \quad\left(\mathrm{Al}_{2} \mathrm{O}_{3}\right.$-substrate $\left.\alpha_{B}=2-3 \%\right)$ [37]. From these data of $\alpha_{B}$ the frequency dependence appears significant. A correction could be necessary for strong coupling superconductors such as $\mathrm{Pb}$, which show strong phonon trapping. Here volume losses in the junction may not be negligible. In principle an increase of the phonon splitting rate with frequency is to be expected from density of phonon state arguments.

The observation of phonon resonances in thin Aljunction films [37] gives evidence for the presence of a damping mechanism in the boundary. Without damping the perpendicular transmission (10) to the substrate is $T_{\perp}>0.995$, and no film resonances are to be expected. With damping, $T_{\perp}$ can decrease to about $T_{\perp} \approx 0.8$, which is sufficient for film resonance observation.

Further indication for boundary losses is the increase of the ballistic phonon yield with polishing quality. In helium the averaged values for mechanical, chemical and sputter polish respectively are $\bar{\alpha}_{m p, \mathrm{He}}=3.4 \%$; $\bar{\alpha}_{c p, \mathrm{He}}=4.7 \% ; \bar{\alpha}_{i p, \mathrm{He}}=5.1 \%$. In vacuum we observed $\alpha_{m p,+o_{2}}=8.8 \% ; \bar{\alpha}_{m p}=9.2 \%, \quad \bar{\alpha}_{c p}=9.5 \% ; \bar{\alpha}_{i p,+o_{2}}=$ $11.2 \%$.

In some vacuum experiments with mechanical polish and in all experiments with sputter polish the tin films have been evaporated with oxygen background where film volume losses can reduce the yield up to a factor of 0.7 compared to the oxygen-free evaporation (see Sect. 4.1). Thus, we expect a further increase in the yield if experiments can be performed without oxygen background evaporation on sputter polished Si substrates. 


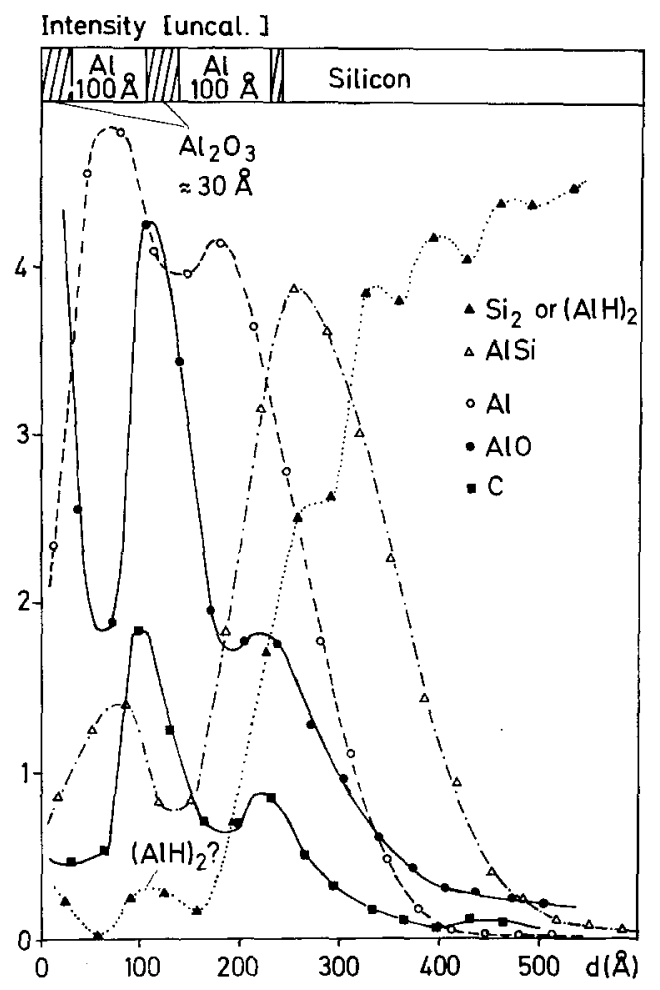

Fig. 6. Dynamic SIMS analysis of the layer structure of an Aljunction on silicon. Notice the existence of carbon $\left(C_{x} H_{y}\right)$ in the boundary to the substrate. The layer structure is shown on top of the figure

We also observed that better polishing quality improved the adhesion of the films to the substrate. This raises the question whether the phonon thermalization originates mainly from inelastic coupling in connection with a weak mechanical film bonding by a layer of impurity atoms. Figure 6 shows the results of a dynamic SIMS-analysis of an Al-junction on silicon. From the Al- and AlO-intensity thickness dependence the layer structure of the junction is well resolved. The carbon curve indicates the presence of carbonhydrates in the junction-substrate interface (pump oil) and with regard to the AlO-curve the presence of $\mathrm{H}_{2} \mathrm{O}$ is possible. The existence of $2 \Delta$ phonon boundary losses by impurity layers would also explain the only slight rise of phonon yield with polishing quality, since it is difficult in our sample preparation to prevent a $5 \AA-20 \AA$ thick layer of extraneous atoms. In addition, it is expected that surface imperfections of the crystal and the superconducting film contribute to the phonon splitting.

We note that these nonideal interface properties and the corresponding anharmonic loss or splitting processes of the observed strength do not lead to more than $\pm 20 \%$ deviations of the total phonon transmission rate at solid-solid interfaces compared to the acoustical model. This is consistent with phonon transmission experiments and calculations $[38,39,7]$. The physical reason for the comparatively small influence of phonon boundary splitting on the total phonon transmission is due to the fact that phonon splitting is a dissipative process and enters the characteristic acoustic boundary impedance as imaginary term. In solid-solid interfaces the real part of the impedance is larger than the imaginary "loss" term. This does not hold at the liquid-solid interface where phonon boundary losses, i.e. phonon splitting, may be responsible for large deviations from the simple acoustic model of phonon transmission, as experimentally found in the Kapitza resistance of the solid-liquid He boundary.

In conclusion, we would like to point out that in addition to the identification of the interface loss processes as main origin of the limited phonon yield our results also demonstrate the validity of the models for phonon generation, propagation and detection used in the calculations of the present work.

We wish to thank W. Forkel, H. Kinder, K. Laßmann, R. Rolcke and M. Welte for stimulating discussions, especially $O$. Weis for supplying Sn-Si phonon transmission data and $\mathrm{K}$. Wittmaak for the SIMS analysis. Financial support by the Deutsche Forschungsgemeinschaft is gratefully acknowledged.

\section{References}

1. Eisenmenger, W., Dayem, A.H.: Phys. Rev. Lett. 18, 125 (1967)

2. Eisenmenger, W.: In: Tunneling Phenomena in Solids (ed. E. Burstein, S. Lundquist). New York: Plenum Press 1969

3. Eisenmenger, W.: In: Physical Acoustics, Bd. XII, 1976 (ed. W.P. Mason and R. Thurston)

4. Eisenmenger, W., Laßmann, K., Trumpp, H.J., Krauß, R.: Journ. of Appl. Phys. 11, 307 (1976)

5. Eisenmenger, W., Laßmann, K., Trumpp, H.J., Krauß, R.: Appl. Phys. 12, 163 (1977)

6. Rothwarf, A., Taylor, B.N.: Phys. Rev. Lett. 19, 27 (1967)

7. Trumpp, H.J., Laßmann, K., Eisenmenger, W.: Phys. Lett. $41 \mathrm{~A}$, $431(1972)$

8. Trumpp, H.J., Epperlein, P.W., Laßmann, K.: Journal de Physique C4 suppl. au No 10, C-29 (1972)

9. Kinder, H., Laßmann, K., Eisenmenger, W.: Phys. Lett. 31 A, 475 (1970)

10. Dayem, A.H., Wiegand, J.J.: Phys. Rev. B5, 4390 (1972)

11. Welte, M., Laßmann, K., Eisenmenger, W.: Journal de Physique 33, C4-25 (1972)

12. Forkel, W., Welte, M., Eisenmenger, W.: Phys. Rev. Lett. 31, $215(1973)$

13. Welte, M.: Thesis, Phys. Inst. Univ. Stuttgart, 1976 (unpublished)

14. Kinder, H.: Phys. Rev. Lett. 28, 1564 (1972)

15. Trumpp, H.J.: Thesis, Phys. Inst., Univ. Stuttgart, 1976 (unpublished)

16. Little, W.A.: Can. Journ. Phys. 37, 334 (1959)

17. Khalatnikow, I.M.: Zh. éksp. teor. Fiz. 22, 687 (1952)

18. Taylor, B., Maris, H.J., Elbaum, C.: Phys. Rev. Lett. 23, 416 (1969) 
19. Elbaum, C.: Intern. Conf. on Phonon Scattering in Solids (ed. H.J. Albany). 3-6 July 1972, Paris

20. Rösch, F., Weis, O.: Z. Physik B25, 115 (1976)

21. Weis, O.: Z. Angew. Phys. 26, 325 (1969)

22. Orbach, R., Vredevoe, L.A.: Physics 1, 91 (1964)

23. Klemens, P.G.: J. Appl. Phys. 38, 4573 (1967)

24. Holland, M.G.: IEEE Transactions on Sonics and Ultrasonics 1 (Suppl. 15), 18 (1968)

25. Bobetic, V.M.: Phys. Rev. 136, 1535 (1964)

26. Dayem, A.H., Miller, B.I., Wiegand, J.J.: Phys. Rev. B3, 2949 (1971)

27. Weis, O.: Private Communication

28. Kapitza, P.L.: Zh. éksp, teor. Fiz. 11, 1 (1941), J. Phys. Moscow 4, 181 (1941)

29. Toombs, G.A., Challis, L.J.: Journ. Phys. C4, 1085 (1970)

30. Haug, H., Weiss, K.: Phys. Lett. 40 A, 19 (1972)

31. Vuorio, M.: Journ. of Low Temp. Phys. 10, 781 (1973)

32. Vuorio, M.: Journ. Phys. C5, 1216 (1972)

33. Peterson, R.E., Anderson, A.C.: Journ. of Low Temp. Phys. 11 639 (1973)
34. Böhm, K.: Diplomarbeit, Phys. Inst. Univ. Stuttgart 1975 (unpublished)

35. Day, W.: Journal de Physique, suppl au No 10, C4-65 (1972)

36. Rolcke, R.: Thesis, Phys. Inst., Univ. Stuttgart, 1977 (unpublished)

37. Forkel, W.: Thesis, Phys. Inst., Univ. Stuttgart, 1977 (unpublished)

38. Herth, P., Weis, O.: Z. Angew. Phys. 29, 101 (1970)

39. Kappus, W., Weis, O.: Appl. Phys. 44, 1947 (1973)

H.J. Trumpp

W. Eisenmenger

Physikalisches Institut

der Universität Stuttgart

Pfaffenwaldring 57

D-7000 Stutgart 80

Federal Republic of Germany 